\title{
The interaction of Airy waves and solitons in the three-wave system
}

\author{
Thawatchai Mayteevarunyoo ${ }^{1}$ and Boris A. Malomed ${ }^{2,3}$ \\ ${ }^{1}$ Department of Telecommunication Engineering, Mahanakorn University of Technology, Bangkok 10530, Thailand \\ ${ }^{2}$ Department of Physical Electronics, School of Electrical Engineering, \\ Faculty of Engineering, Tel Aviv University, Tel Aviv 69978, Israel \\ ${ }^{3}$ Laboratory of Nonlinear-Optical Informatics, ITMO University, St. Petersburg 197101, Russia
}

\begin{abstract}
We employ the generic three-wave system, with the $\chi^{(2)}$ interaction between two components of the fundamental-frequency (FF) wave and second-harmonic (SH) one, to consider collisions of truncated Airy waves (TAWs) and three-wave solitons in a setting which is not available in other nonlinear systems. The advantage is that the single-wave TAWs, carried by either one of the FF component, are not distorted by the nonlinearity and are stable, three-wave solitons being stable too in the same system. The collision between mutually symmetric TAWs, carried by the different FF components, transforms them into a set of solitons, the number of which decreases with the increase of the total power. The TAW absorbs an incident small-power soliton, and a high-power soliton absorbs the TAW. Between these limits, the collision with an incident soliton converts the TAW into two solitons, with a remnant of the TAW attached to one of them, or leads to formation of a complex TAW-soliton bound state. At large velocities, the collisions become quasi-elastic.
\end{abstract}

\section{INTRODUCTION}

Airy waves represent a universal transmission mode in linear media governed by linear Schrödinger equations, which makes it possible to send free beams along self-bending trajectories [1. While the full Airy mode has a divergent total norm, it was predicted that its finite-norm version, i.e., a truncated Airy wave, TAW, supports similar curvilinear transmission 22. The propagation of TAWs was demonstrated, theoretically and experimentally, in various settings in optics [2]-[9], plasmonics [10-[12, electron beams [13], matter waves [14, gas discharge [15], and water waves [16].

While the Airy waves and their truncated version are eigenmodes of the linear transmission, the propagation of their counterparts distorted by the self-interaction was also studied in media with cubic [17]-223 and quadratic (alias $\left.\chi^{(2)}\right)$ 24-27. nonlinearities. In the latter case, the TAW launched as the fundamental-frequency (FF) wave gives rise to two-color beams, generating the second-harmonic $(\mathrm{SH})$ component via the $\chi^{(2)}$-mediated upconversion.

Because TAWs feature multi-lobe structures [2, while stable eigenmodes of nonlinear media are single-hump solitons, a natural outcome of the nonlinear transformation may be shedding of solitons by the TAW under the action of the cubic nonlinearity [16, 18. A somewhat similar outcome of the evolution is downconversion of a TAW launched, as an exact linear SH mode, into a set of $\chi^{(2)}$ solitons, initiated by the parametric instability of the SH wave seeded by FF perturbations, in one- 28 and two-dimensional 29 settings alike.

Another natural possibility in nonlinear media is interaction between TAWs, as well as between them and solitons. Such interactions were theoretically studied in cubic [30]-32, [8] and photorefractive [33] media, in systems with nonlocal nonlinearities [34, 35, and in a model of coherent atomic media [36. In all these cases, the situation is rather tricky, as the nonlinearity distorts the TAW even without its interaction with another mode. In the present work, we aim to propose a system which makes it possible to study interactions between undistorted stable Airy waves, as well as their interactions with stable solitons. This model is based on the nondegenerate, i.e., three-wave, system with quadratic couplings between two FF components and the SH wave (unlike the degenerate version, in which the $\chi^{(2)}$ terms couple the SH to the single FF component, that was considered in previous works dealing with Airy waves in quadratic media [24-[27]). The two FF complex wave fields, $u$ and $v$, usually represent orthogonal polarizations of the waves carried by the $\mathrm{FF}$, while the $\mathrm{SH}$ field, $w$, is represented by a single polarization. In the spatial domain, the three-wave system, which corresponds to the so-called Type-II $\chi^{(2)}$ interaction in a planar waveguide, is modeled by the well-known propagation equations, written in the scaled form corresponding to the paraxial approximation [37. 39]:

$$
\begin{aligned}
i u_{z}+b u+\frac{1}{2} u_{x x}+v^{*} w & =0 \\
i v_{z}-b v+\frac{1}{2} v_{x x}+u^{*} w & =0, \\
2 i w_{z}-q w+\frac{1}{2} w_{x x}+u v & =0,
\end{aligned}
$$

where $z$ and $x$ are the propagation distance and transverse coordinate, real coefficients $b$ and $q$ represent the birefringence and mismatch parameters, respectively, and $*$ stands for the complex conjugate field. The system conserves the 
total power (alias the Manley-Rowe invariant, or total norm),

$$
P=\int_{-\infty}^{+\infty}\left(|u|^{2}+|v|^{2}+4|w|^{2}\right) d x \equiv P_{u}+P_{v}+P_{w}
$$

the total momentum,

$$
M=i \int_{-\infty}^{+\infty}\left(u u_{x}^{*}+v v_{x}^{*}+2 w w_{x}^{*}\right) d x
$$

and the Hamiltonian,

$$
H=\int_{-\infty}^{+\infty}\left(\left|u_{x}\right|^{2}+\left|v_{x}\right|^{2}+\left|w_{x}\right|^{2}+b|v|^{2}-|u|^{2}+q|w|^{2}+u^{*} v^{*} w+u v w^{*}\right) d x
$$

Scaled variables $x$ and $z$ are measured, respectively, in units of the characteristic size of $\chi^{(2)}$ patterns (such as solitons), which is $l \sim 50 \mu \mathrm{m}$ [37]-39], and the respective diffraction length, $\Delta z \sim(2 \pi / \lambda) l^{2} \sim 1 \mathrm{~cm}$, for the carrier wavelength $\lambda \sim 1 \mu \mathrm{m}$. Then, $q=1$ and $b=1$ correspond, in physical units, to the mismatch $1 \mathrm{~cm}^{-1}$. As concerns tilted ("moving") solitons (e.g., ones with slopes $1 / 4$ and $1 / 3$ in the scaled variables, which are shown below in Fig. $3(\mathrm{a})$ and $8(\mathrm{a})-12(\mathrm{a})$, respectively), their slope, as measured in real coordinates, is smaller by a factor $\sim 0.01$.

Actually, parameter $b$ can be eliminated from Eqs. (1) by means of a substitution,

$$
\{u(x, z), v(x, z)\} \equiv\left\{e^{i b z} \tilde{u}(x, z), e^{-i b z} \tilde{v}(x, z)\right\}
$$

On the other hand, as concerns soliton solutions considered below, fixing propagation constants of the $u$ and $v$ waves and varying $b$, it is possible to analyze the change of the solutions following the variation of the difference between full propagation constants of the two FF components, see Fig. 2(a) below.

An advantage offered by system (1) is that it admits exact stable TAW solutions, with $v=w=0$ and the same form of the $u$ component as in the single linear Schrödinger equation [2]:

$$
\begin{array}{r}
u_{\mathrm{TAW}}(x, z)=u_{0} \operatorname{Ai}\left(\alpha x-\frac{\alpha^{4}}{4} z^{2}+i \aleph \alpha^{2} z\right) \\
\times \exp \left(-\frac{i}{12} \alpha^{6} z^{3}+\frac{i}{2} \alpha^{3} x z\right) \\
\times \exp \left(\aleph \alpha x-\frac{1}{2} \aleph \alpha^{4} z^{2}+\frac{i}{2} \aleph^{2} \alpha^{2} z+i b z\right),
\end{array}
$$

where $\mathrm{Ai}$ is the standard Airy function, while $u_{0}, \alpha$, and $\aleph$ are positive constants that define, respectively, the amplitude, intrinsic scale, and truncation of the Airy wave, as seen from the form of the initial condition which generates the TAW solution:

$$
\left.u(x)\right|_{z=0}=u_{0} \operatorname{Ai}(\alpha x) \exp (\aleph \alpha x),\left.v(x)\right|_{z=0}=\left.w(x)\right|_{z=0}=0 .
$$

The total momentum (3) of real waveform (7) is zero, while its total power is [2]

$$
P_{\mathrm{TAW}}=\frac{u_{0}^{2}}{\sqrt{8 \pi \aleph} \alpha} \exp \left(\frac{2}{3} \aleph^{3}\right)
$$

A symmetric counterpart of the TAW, with the center shifted by $x_{0}$ and inverted orientation (which is necessary for the consideration of collisions between the TAWs, see below) can be obtained from Eq. (6) by setting

$$
v_{\mathrm{TAW}}(x, z)=u_{\mathrm{TAW}}\left(x-x_{0}, z, b \rightarrow-b, \alpha \rightarrow-\alpha\right), u=w=0
$$

(note that this transformation does not change the sign of truncation parameter $\aleph$ ).

In previous work 28, TAW was taken as the exact solution of the linear version of the equation for the SH field, $w$, in system (1), while setting $u=v=0$. The so introduced TAW was subject to the parametric instability seeded by small perturbations in the $u$ and $v$ fields, which leads to spontaneous downconversion of the TAW into a cluster of $\chi^{(2)}$ solitons and additional radiation jets (in fact, the degenerate two-wave version of the $\chi^{(2)}$ system was considered in that case, but the TAW state in the SH field remains unstable in the three-wave system as well). In the present case, it is easy to demonstrate, considering small perturbations in the $v$ and $w$ fields, that the exact TAW solution (6) 
in the $u$ field, as well as its $v$-field counterpart, given by Eq. (9), are stable against perturbations (this conclusion is corroborated by direct simulations of the perturbed evolution of the TAWs, which are not displayed here, as they do not show anything essentially new). Thus, the system (1) suggests a novel possibility to consider nonlinear interaction between two stable undistorted TAWs, namely, ones given by Eqs. (6) and (9). Further, the three-wave system gives rise to three-component solitons [40, 41. The Galilean invariance of the system makes it possible to boost the solitons to an arbitrary velocity (actually, moving solitons are tilted spatial beams), hence the same system makes it possible to study collisions of the three-component solitons with the stable TAW created in one FF component, as well as soliton-soliton collisions.

The rest of the paper is organized as follows. The three-wave solitons of system (1), which are available in a particular analytical form, and in a numerical form in the general case, are introduced in Section II. Typical examples of elastic and inelastic collisions between moving stable solitons are presented in Section II too. Collisions between the TAW and its mirror-image counterpart, given by Eqs. (6) and (9), are considered by means of direct simulations in Section III. The analysis is continued in Section IV, which reports results of systematic simulations of collisions between moving three-wave solitons and TAWs. The paper is concluded by Section V.

\section{THREE-COMPONENT SOLITONS}

Soliton solutions to Eq. (1) are looked for as

$$
u(x, z)=U_{\mathrm{sol}}(\xi) e^{i k_{1} z+i c x}, v(x, z)=V_{\mathrm{sol}}(\xi) e^{i k_{2} z+i c x}, w(x, z)=W_{\mathrm{sol}}(\xi) e^{i\left(k_{1}+k_{2}\right) z+2 i c x},
$$

where $\xi \equiv x-c z, c$ is the soliton's velocity (actually, the tilt in the spatial domain), $k_{1}$ and $k_{2}$ are two independent propagation constants, which are free parameters of the soliton family, and real functions $U_{\text {sol }}, V_{\text {sol }}$, and $W_{\text {sol }}$ are solutions of the stationary equations:

$$
\begin{gathered}
\frac{1}{2} \frac{d^{2} U}{d \xi^{2}}+V W=\left(k_{1}-b\right) U, \\
\frac{1}{2} \frac{d^{2} V}{d \xi^{2}}+U W=\left(k_{2}+b\right) V, \\
\frac{1}{2} \frac{d^{2} W}{d \xi^{2}}+U V=\left[2\left(k_{1}+k_{2}\right)+q\right] W .
\end{gathered}
$$

Obvious conditions necessary for the existence of exponentially localized solutions to these equations are

$$
-k_{2}<b<k_{1}, k_{1}+k_{2}>-q / 2 .
$$

Following Ref. [40, it is straightforward to find particular exact solutions of Eq. (11), with mutually symmetric $U$ and $V$ components, for $q<0$ :

$$
\begin{gathered}
k_{1}=b-(q / 3), k_{2}=-b-(q / 3), \\
U(x)=V(x)=-W(x)=(q / 2) \operatorname{sech}^{2}(\sqrt{-q / 6} \xi) .
\end{gathered}
$$

The total power (2) and momentum (3) of the exact soliton solutions given by Eqs. (10) and (14) are

$$
P=2 \sqrt{-6 q^{3}}
$$

and $M=c P$, the latter relation between $M$ and $P$ being valid for all solitons (not only for exact ones given by Eqs. (13) and (14)).

Symmetric solitons with $U=V$ are tantamount to their well-known counterparts in the degenerate (two-component) version of the $\chi^{(2)}$ system; in particular, exact solutions given by Eqs. 13) and (14) are equivalent to the KaramzinSukhorukov soliton solutions of the degenerate system [37-39]. However, stability of the symmetric solitons may be different in the three-wave system - in particular, three-wave solitons with the symmetry between $U$ and $V$ should be stable against symmetry-breaking perturbations.

General three-component soliton solutions to Eq. (11), without assuming the symmetry between the two FF components, can be easily constructed by means of the Newton's method. Numerical computations were carried out in the domain of $|x| \leq 2^{11}$ with zero boundary conditions, discretized by $2^{13}$ grid points, achieving a limitation of the 


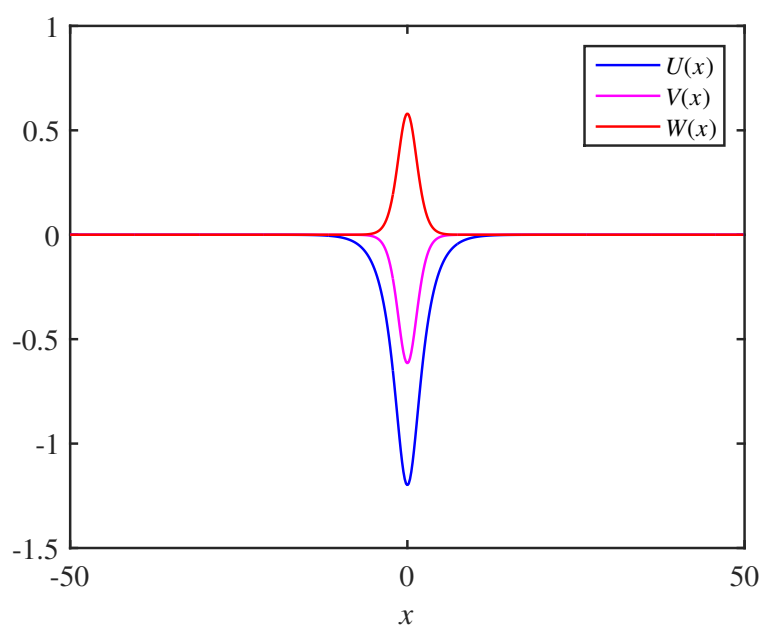

FIG. 1: (Color online) A generic example of a stable three-component soliton, with unequal $u$ - and $v$-components, found as a solution of Eq. (11) (with $\xi$ replaced by $x$ ) for parameters $q=-1, b=0.2$, and propagation constants $k_{1}=1 / 3$ and $k_{2}=2 / 3$. The same solution may be taken with opposite signs of $U$ and $V$.

residual relative error $<10^{-10}$. The Newton's method was implemented for fixed $k_{1}$ and $k_{2}$, starting from the simplest input which was suggested by the structure of the exact symmetric solution (14), viz., $u_{\text {in }}=v_{\text {in }}=-w_{\text {in }}=u_{0} \exp \left(-x^{2}\right)$, with some constant $u_{0}$. A typical example of the so found asymmetric solitons is displayed in Fig. 1.

Families of generic asymmetric solitons are represented by dependences $P(b)$ for several values of $q$, and $P(q)$ for several values of $b$, which are displayed in Fig. 2 for fixed values of both propagation constants, $k_{1}=1 / 3$ and $k_{2}=2 / 3$. In particular, conditions (12) determine the left and right edges of the existence region of the soliton families in Fig. 2(a), which are, respectively, $b=-k_{2}=-2 / 3$ and $b=k_{1}=1 / 3$, as well as the left edge in Fig. 2(b), $q=-2\left(k_{1}+k_{2}\right)=-1$. Further, the nearly linear form of the right branch of the $P(q)$ dependence in Fig. 2(b) can be easily explained by the cascading approximation, which assumes that the diffraction term (second derivative) may be neglected in the equation for $W$ [37]-39]. Indeed, eliminating the $\mathrm{SH}$ field under this assumption,

$$
W \approx U V / Q, Q \equiv 2\left(k_{1}+k_{2}\right)+q,
$$

one arrives at a system of equations for $U$ and $V$,

$$
\begin{aligned}
& \frac{1}{2} \frac{d^{2} U}{d \xi^{2}}+\frac{1}{Q} V^{2} U=\left(k_{1}-b\right) U, \\
& \frac{1}{2} \frac{d^{2} V}{d \xi}+\frac{1}{Q} U^{2} V=\left(k_{2}+b\right) V .
\end{aligned}
$$

It is obvious that the total norm of solutions of system (17) scales $\sim Q$, i.e., as a linear function of $q$.

As shown below, collisions between TAWs often generate strongly asymmetric solitons, with one FF component being much taller and broader than the other, and a still smaller SH component. It is easy to obtain an approximate form of the strongly asymmetric solitons from Eq. (17). If, for instance, the $U$ and $V$ components are the large and small ones, the effective small-amplitude narrow potential in the first equation in system [17, $-Q^{-1} V^{2}(x)$, may be approximated by a delta-function. This approximation yields the following result:

$$
U(x) \approx \sqrt{Q\left(k_{2}+b\right)} \exp \left(-\sqrt{2\left(k_{1}-b\right)}|x|\right), V^{2}(x) \approx Q \sqrt{2\left(k_{1}-b\right)} \delta(x),
$$

with the respective powers

$$
P_{u}=\frac{Q\left(k_{2}+b\right)}{\sqrt{2\left(k_{1}-b\right)}}, P_{v}=Q \sqrt{2\left(k_{1}-b\right)},
$$

while the power of the $\mathrm{SH}$ component is negligible in this approximation. The underlying condition of the dominance of the $U$ component implies $P_{v} / P_{u} \ll 1$, i.e., $k_{1}-b \ll k_{2}+b$. The soliton with large $V$ and small $U$ components can be obtained from here by substitution $k_{2} \rightleftarrows k_{1}, b \rightarrow-b$. A detailed consideration demonstrates that Eq. (19) and 


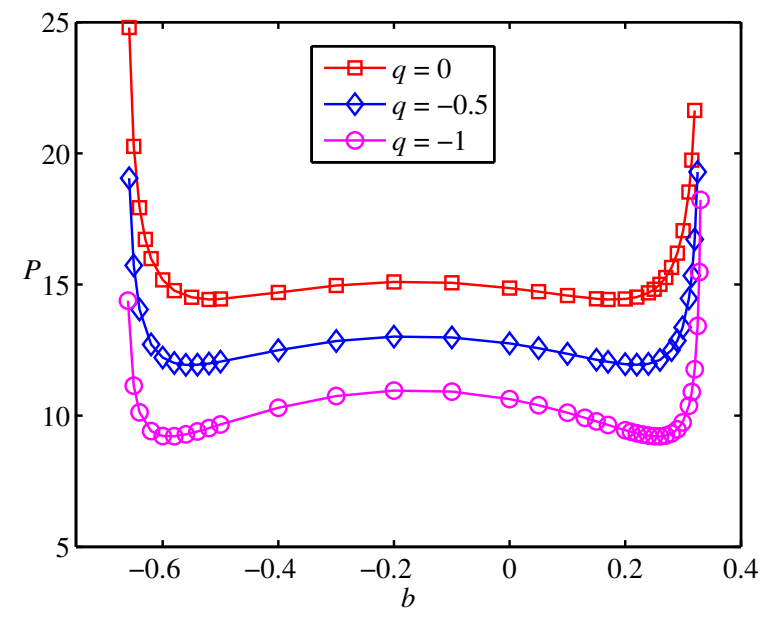

(a)

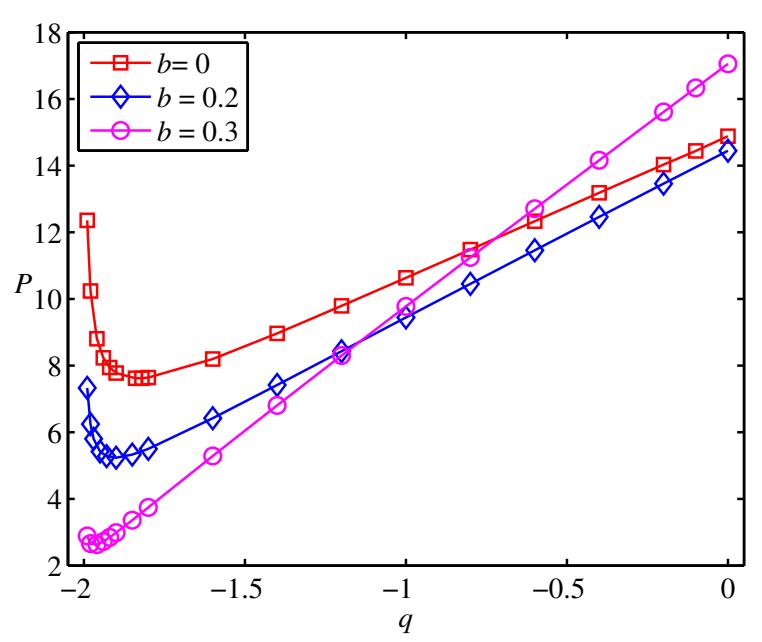

(b)

FIG. 2: (Color online) (a) The total power $P$ of the three-wave solitons versus the birefringence coefficient, $b$, for three different values of mismatch $q$. (b) $P$ versus $q$ for three different values of $b$. In both panels, the propagation constants are fixed, $k_{1}=1 / 3$ and $k_{2}=2 / 3$, see Eq. [11.

its counterpart, corresponding to the swap between $U$ and $V$, correctly approximate the right and left peaks of the dependences displayed in Fig. 2(a).

Stability of the three-component solitons was tested by systematic simulations of their perturbed evolution, using the split-step fast-Fourier-transform algorithm. The simulations were typically performed in the propagation interval $0 \leq z \leq 500$, which roughly corresponds to 20 diffraction lengths of the typical soliton displayed in Fig. 1. In the course of the simulations, the conservation of the total power (2) and momentum 3 holds with relative accuracy no worse than $10^{-5}$. As a result, it has been concluded that the entire family of exact solitons (13), (14) is stable, as well as all the asymmetric solitons found in the numerical form.

Before proceeding to the consideration of collisions of solitons with TAWs, and collisions of TAWs between themselves, it is relevant to briefly consider collisions between moving solitons. The simulations demonstrate a situation typical for interactions of solitons in nonintegrable systems [2]: collisions between relatively slow solitons lead to their merger into a single excited localized state, which is accompanied by the emission of small-amplitude dispersive waves, while fast solitons interact quasi-elastically, passing through each other, as shown, in terms of the evolution of field $|u(x, z)|$, in Figs. 3(a) and (b), respectively (the evolution of the other fields, $v$ and $w$, shows a similar picture). For values of parameters fixed in this figure, $b=0$ and $q=-1$, the critical velocity which separates the inelastic and elastic collisions of the solitons moving with velocities $\pm c$ is $c_{\mathrm{cr}} \approx 0.245$.

\section{COLLISIONS BETWEEN COUNTER-PROPAGATING AIRY WAVES}

As mentioned above, the three-wave system makes it possible to consider collisions between undistorted TAWs, such as one given by Eq. (6) and the counterpropagating one (9). The result of the collision strongly depends on the strength of the nonlinearity, which is determined by amplitude $u_{0}$ in Eq. (6). This is illustrated, in a generic form, in Figs. 4.7 by a set of results generated for the pair of the TAWs with $\alpha= \pm 0.2, \aleph=0.1$, launched with initial separation $X=40$ between their centers, while parameters in Eq. (1) are taken as $b=q=0$. In principle, the outcome of the collision may also depend on a phase shift between the two mutually symmetric TAWs [31; however, the simulations demonstrate that this effect is inconspicuous, due to the fact that the variation of the phase across the TAWs given by Eqs. (6) and (9) becomes larger than the phase shift between them.

First, in the quasi-linear regime, which corresponds to a relatively small amplitude, $u_{0}=1$, Fig. 4 demonstrates transformation of the interacting Airy waves into a complex multi-peak pattern, in which individual peaks may be roughly traced backs to lobes of the colliding TAWs. The increase of the amplitude from $u_{0}=1$ to 3 leads to coagulation of many peaks into fewer taller ones, due to the nonlinear self-attraction, as seen in Fig. 5 . The trend continues with further increase of $u_{0}$, producing the cluster of peaks displayed in Fig. 6 for $u_{0}=5$. In the latter case, detailed consideration definitely confirms that each isolated tall peak may be identified as a stable three-wave solitona quiescent symmetric one at the center, and eight strongly asymmetric moving solitons, which may be approximated 


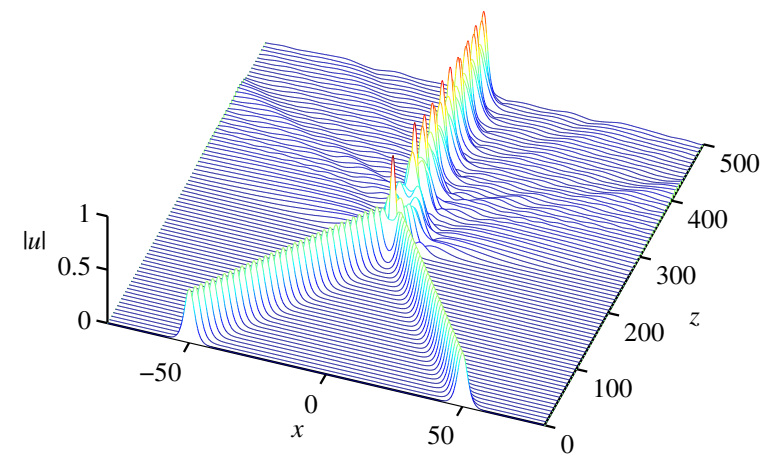

(a)

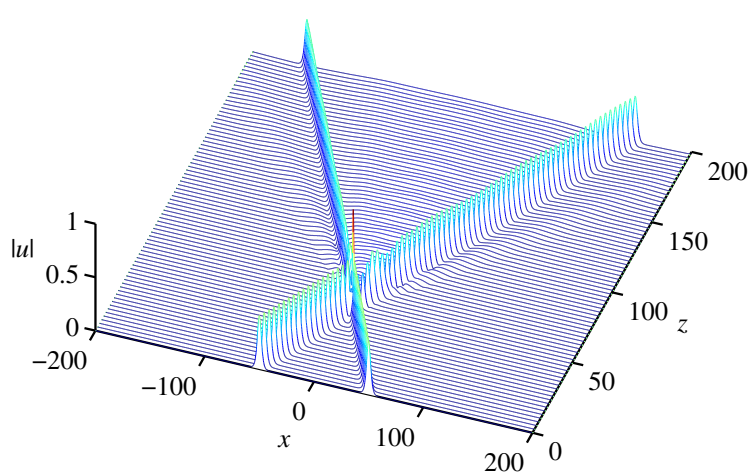

(b)

FIG. 3: (Color online) (a) Merger of three-wave solitons, given by exact solution (13)-(14), which collide with velocities $c= \pm 0.23$. (b) A quasi-elastic collision of solitons moving with velocities $c= \pm 1$. The initial distance between the solitons is $\Delta x=100 ;$ other parameters are $b=0$ and $q=-1$.

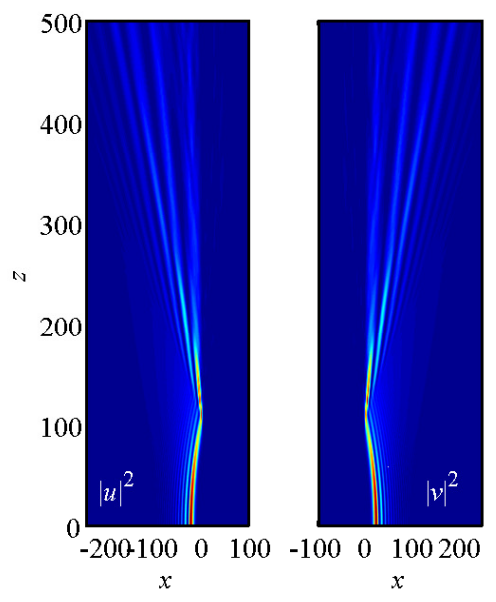

(a)
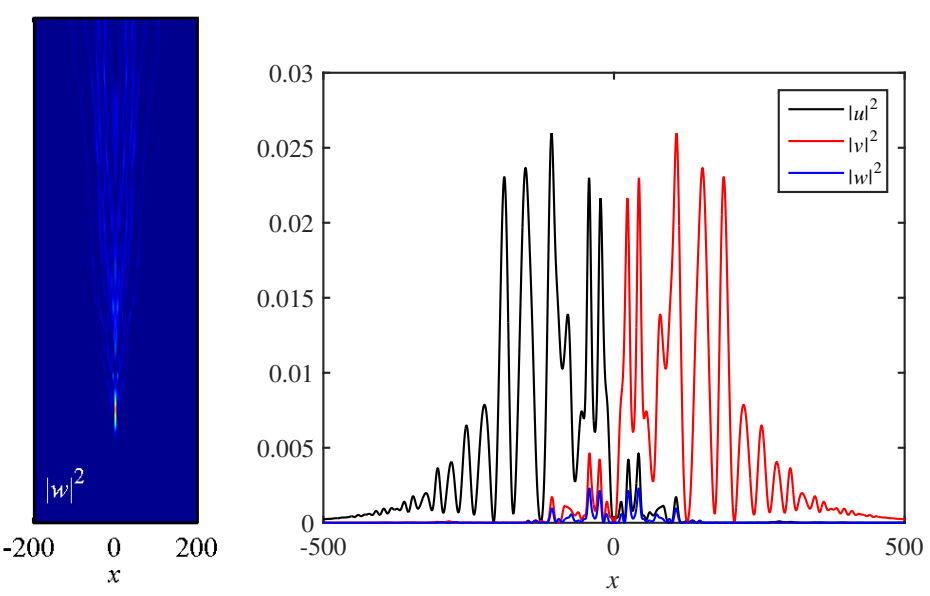

(b)

FIG. 4: (Color online) (a) The collision between two Airy waves, (6) and (9), launched with parameters $\alpha= \pm 0.2, \aleph=0.1, u_{0}=$ 1 , is displayed by means of the evolution of local powers $|u(x, z)|^{2},|v(x, z)|^{2}$, and $|w(x, z)|^{2}$. (b) The final power profiles of fields $u, v$ and $w$ at $z=500$, which demonstrate the transformation of the colliding waves into a complex multi-peak pattern.

by Eqs. 18) and (19), with the Galilean boost $(\sim c)$ applied to them, as per Eq. 10 .

Eventually, under the action of sufficiently strong nonlinearity, the collision of two TAWs leads to the formation of precisely three solitons: the central symmetric one, and a pair of strongly asymmetric left- and right-traveling solitons. This ultimate outcome of the collisions is achieved by increasing the input amplitudes from $u_{0}=5$ in Fig. 6. where nine solitons are still clearly identified in the output, to $u_{0}=6$ (i.e., increasing the total power by $44 \%$ ), as shown in Fig. 7.

It is relevant to note that, in the case of the sufficiently strong nonlinearity, the origin of individual solitons in the output of the collision cannot be traced back to main lobes of the initial TAW profiles. In particular, additional simulations (not shown here in detail) demonstrate that, in the moderately nonlinear situation, the number of generated solitons is essentially affected by varying $\alpha$, i.e., the intrinsic scale of the TAW in Eq. (7), but not by changing parameter $\aleph$, which determines the number of lobes in the waveform (7). 


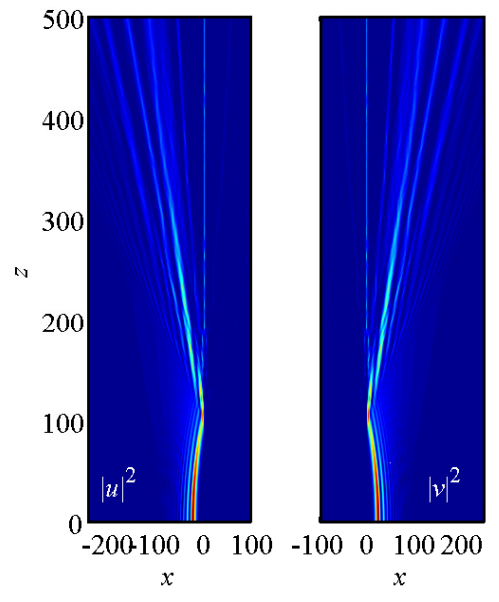

(a)
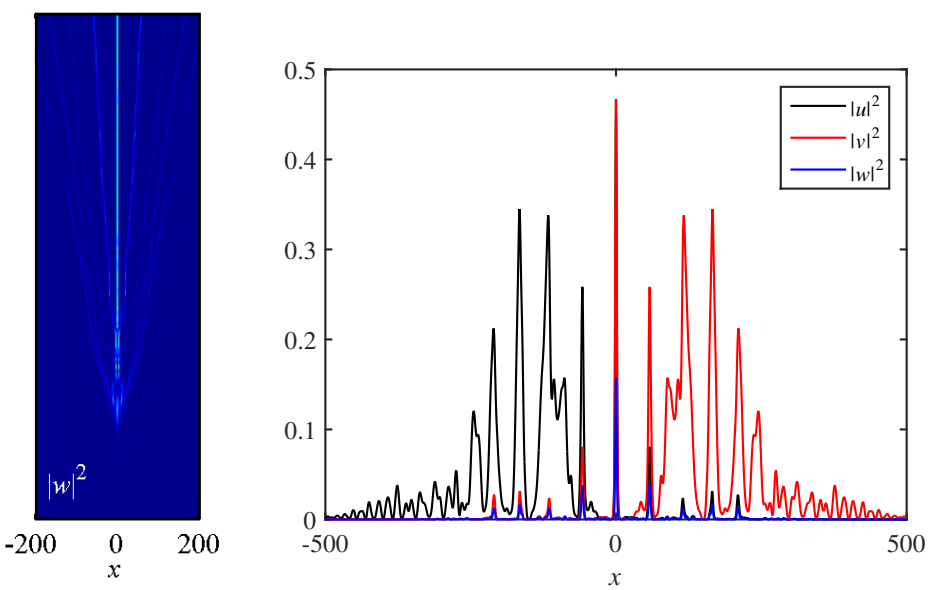

(b)

FIG. 5: (Color online) The same as in Fig. 4 but for $u_{0}=3$. In these plots and similar ones, displayed below for $u_{0}=5$ and 6 in Figs. 6(b) and 7(b), isolated peaks can be identified as three-wave solitons, see the text.

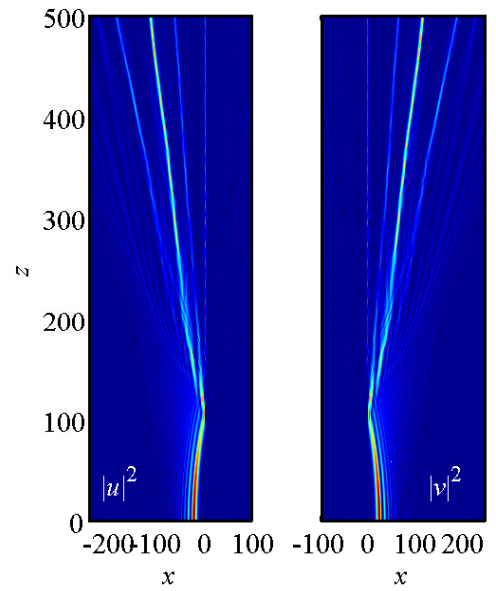

(a)
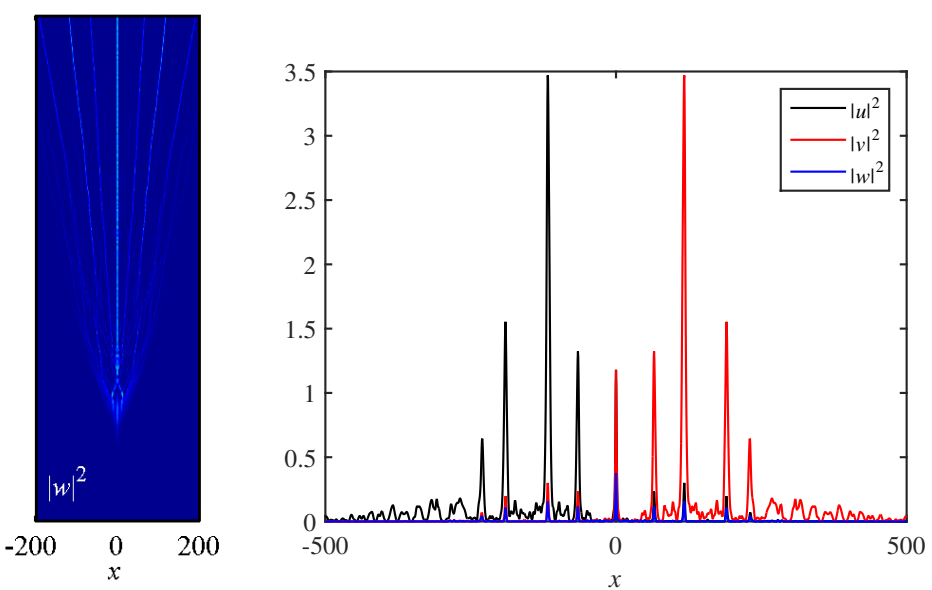

(b)

FIG. 6: (Color online) The same as in Figs. 4 and 5 but for $u_{0}=5$.

\section{AIRY-SOLITON COLLISIONS}

Because both the single-component TAWs and three-component solitons are eigenmodes of the three-wave system, it is natural to study interactions (collisions) between them. To this end, we took the TAW in the form of Eq. (7) with

$$
u_{0}=1, \alpha=\aleph=0.1,
$$

the respective power being $P_{\mathrm{TAW}} \approx 6.29$, as per Eq. $(8)$. The main lobe of this Airy wave has width $L_{\mathrm{FWHM}} \approx 17.1$. Systematic simulations demonstrate that this choice of the parameters adequately represents the generic situation.

The simulations demonstrate that the TAW is a much more fragile object than the soliton, which is not surprising, as the TAW has a complex, hence more vulnerable, structure, and this mode, unlike the soliton, is not adjusted to the action of the nonlinearity. The first series of simulations was performed for collisions of the TAW with symmetric solitons (14), keeping a fixed initial separation between their centers, $\Delta x=100$, and a relatively small soliton's velocity, $c=-0.2$, that makes it possible to observe effects of sufficiently strong interactions (if the velocity is too large, the soliton quickly passes the TAW, which does not allow the system to accumulate interaction effects, as shown 


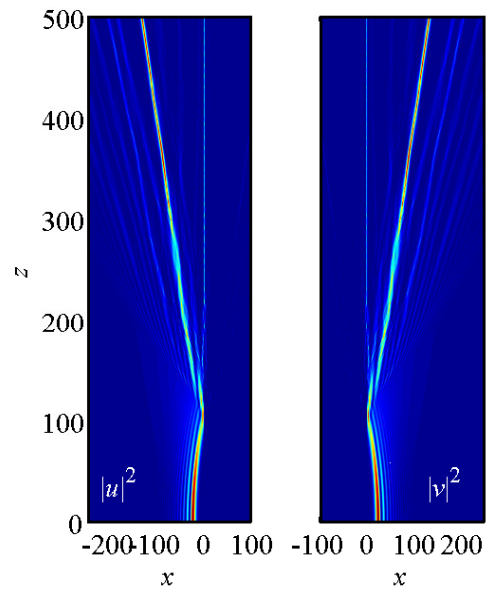

(a)

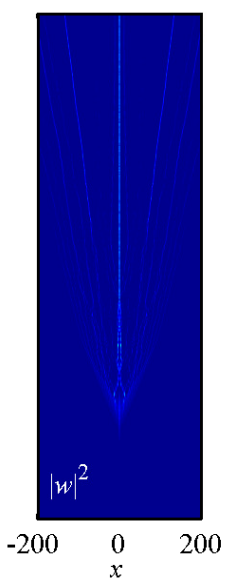

FIG. 7: (Color online) The same as in Figs. 4.6. but for $u_{0}=6$.

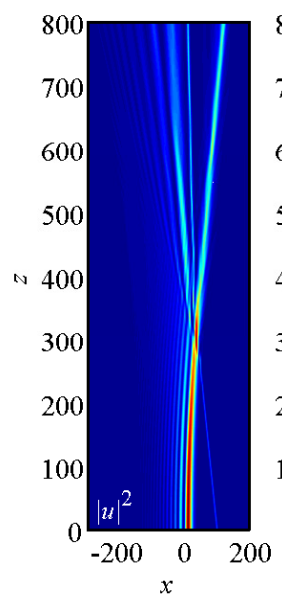

(a)

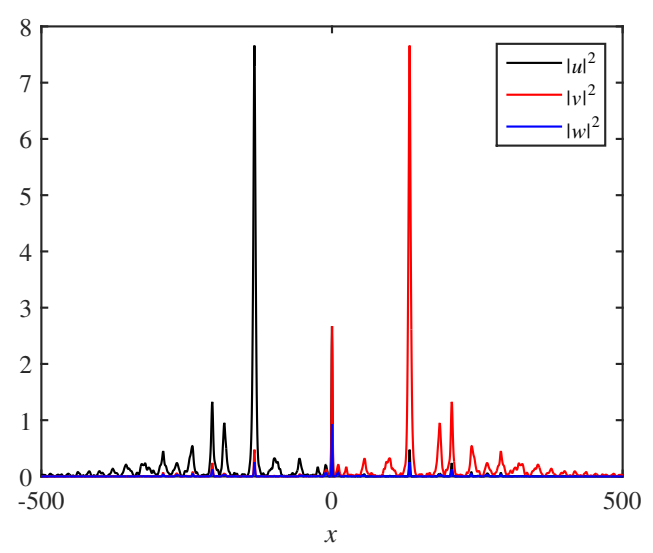

(b)

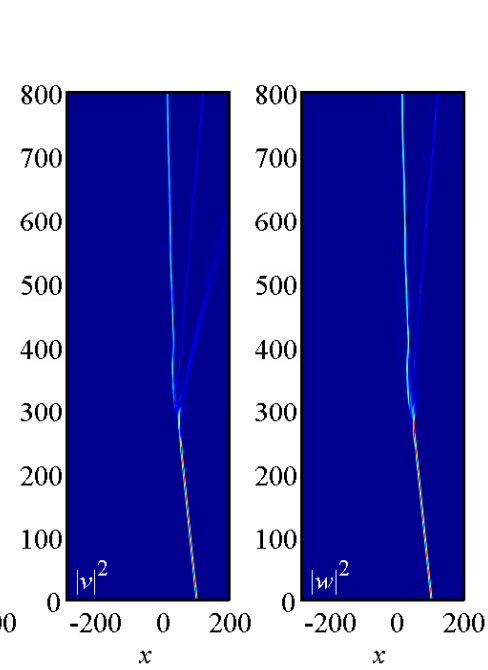

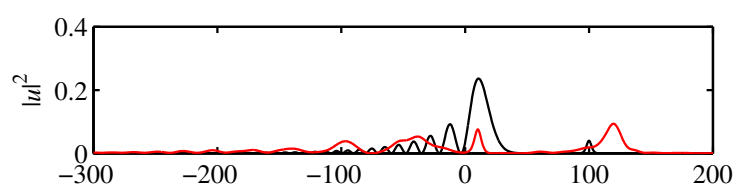
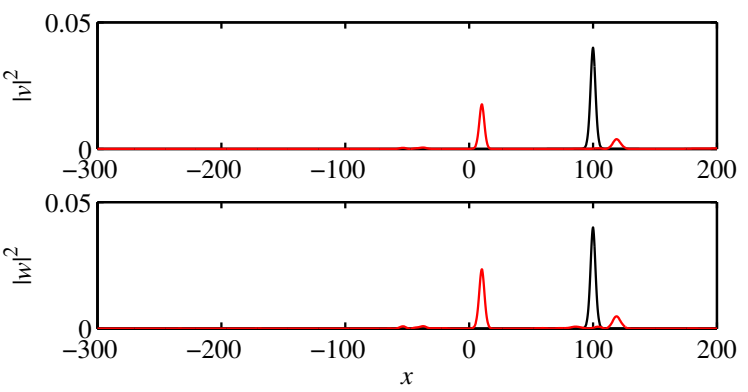

(b)

FIG. 8: (Color online) (a) The collision between the Airy wave with parameters 20 and the symmetric soliton (14), at $q=-0.4$, with velocity $c=-0.2$, is displayed by means of the evolution of local powers $|u(x, z)|^{2},|v(x, z)|^{2}$, and $|w(x, z)|^{2}$. (b) Black and red profiles display, respectively, initial and final power profiles of fields $u, v$ and $w$ at $z=500$, which demonstrate essential disturbance of the Airy wave produced by the collision. Note different vertical scales in the three plots in (b), and in similar plots displayed in subsequent figures below.

below). When the soliton's power $P_{\text {sol }}$, given by Eq. (15), is very small, one may expect that it may be absorbed by the TAW. This happens indeed, for $P_{\text {sol }} \lesssim 0.5$, which corresponds to the soliton's amplitudes $-q \lesssim 0.2$ in Eq. 14). If $-q$ and, accordingly, $P_{\text {sol }}$ become somewhat larger, Fig. 8 demonstrates that the soliton with $-q=0.4$, i.e., $\bar{P}_{\text {sol }} \approx 1.24$ (roughly, $1 / 5$ of $P_{\mathrm{TAW}}$ ), is still absorbed by the TAW, which suffers essential disruption, being on the verge of splitting into solitons.

The fragility of the Airy wave becomes apparent at still larger values of $P_{\text {sol }}$ : the TAW starts to play the role of the reservoir of power, which is split into fragments by the incident soliton. In particular, the soliton carrying $P_{\text {sol }} \approx$ $2.74 \approx 0.44 P_{\mathrm{TAW}}$, which corresponds to $q=-0.68$, breaks the TAW, as shown in Fig. 9 In this case, the outcome of the collision is quite simple: the incident symmetric soliton passes through the TAW, snatching a large share of the power from the Airy wave, and growing to become strongly asymmetric. The so amplified asymmetric soliton emerges with a tail attached on its left side, which may be considered as a remnant of the Airy wave. The remaining part of the initial TAW's power self-traps into another asymmetric soliton, which follows the propagation direction of the original TAW. A qualitatively similar outcome of the collision (which is, thus, a generic one in certain interval of 


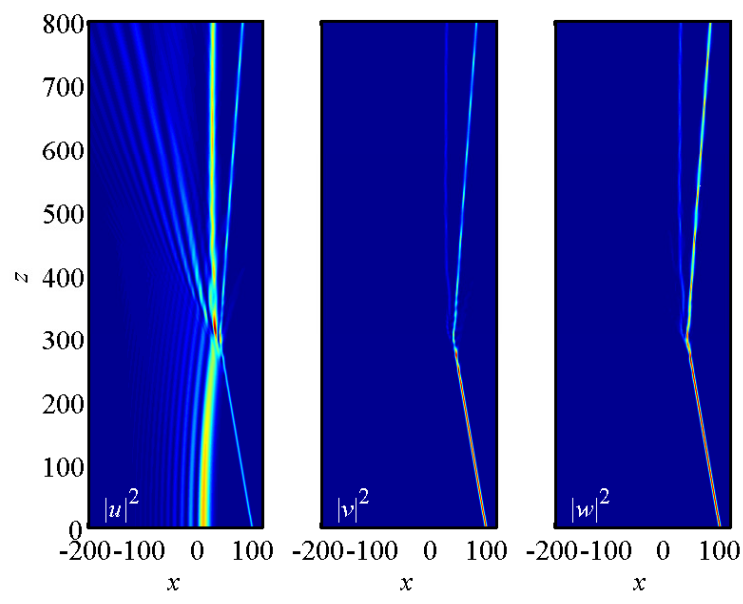

(a)
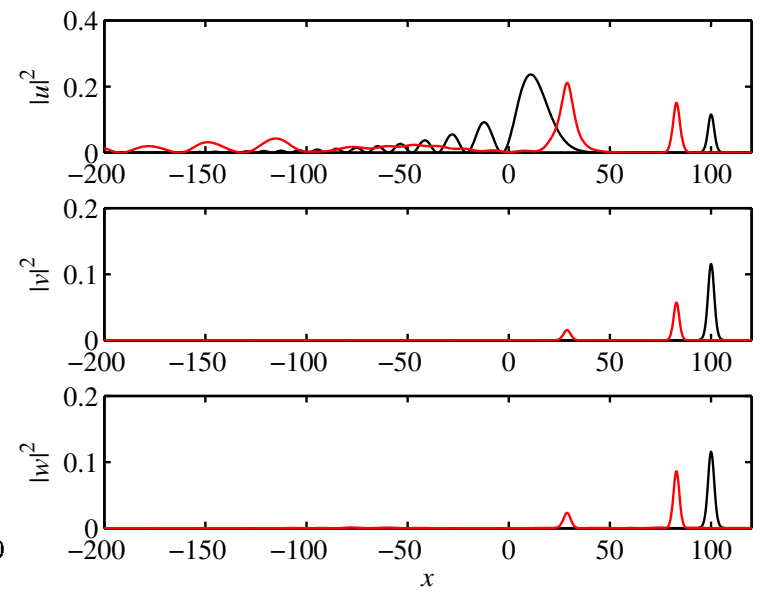

(b)

FIG. 9: (Color online) The same as in Fig. 8 but for $q=-0.68$. In this case, the collision transforms the TAW-soliton pair into a set of two separating solitons, with a remnant of the Airy wave attached to one of them.

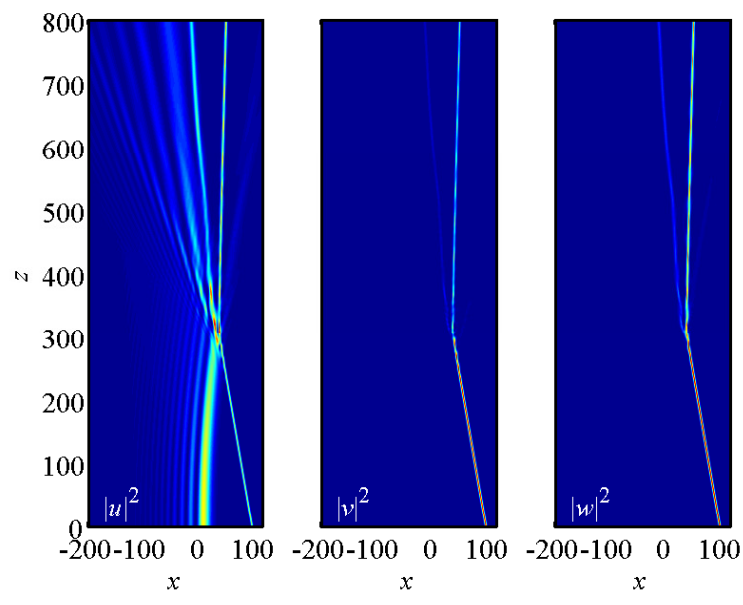

(a)
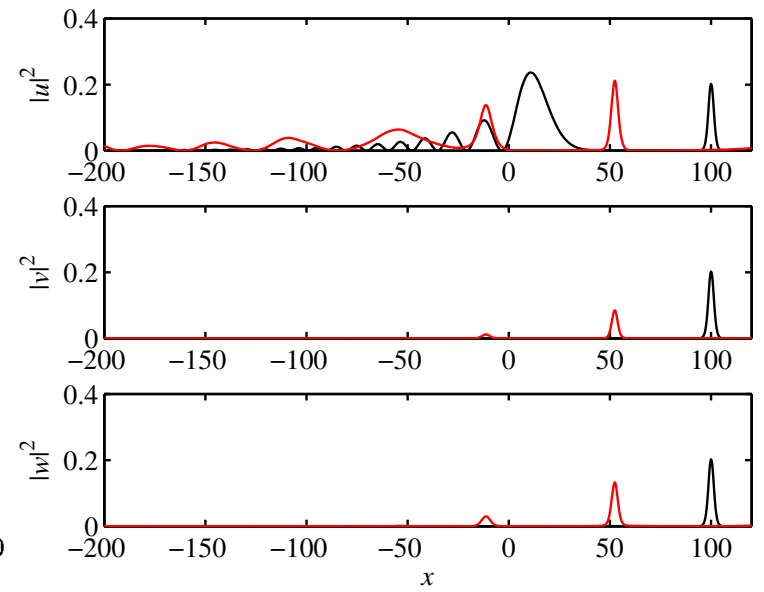

(b)

FIG. 10: (Color online) The same as in Figs. 8 and 9 but for $q=-0.9$.

the values of $\left.P_{\text {sol }}\right)$ is observed at $P_{\text {sol }}=4.18 \approx 0.66 P_{\mathrm{TAW}}$, which corresponds to $q=-0.9$, as seen in Fig. 10

With the increase of $P_{\mathrm{sol}}$ to the value close to $P_{\mathrm{TAW}}$, which corresponds to $q=-1.20$ (in this case, Eq. (8) yields $P_{\text {sol }}=6.44 \approx 1.02 P_{\text {TAW }}$ ), the simulations demonstrate the most complex outcome in Fig. 11 the original TAW and symmetric soliton with nearly equal powers merge into a complex which keeps both the Airy and soliton components discernible, although the latter one becomes asymmetric, and the TAW is essentially distorted too. The merger leads to cancellation of the TAW's and soliton's velocities, so that the bound complex emerges in a nearly quiescent form.

Finally, when $P_{\text {sol }}$ becomes essentially larger than $P_{\text {TAW }}$, viz., at $P_{\text {sol }} \geq 11.71 \approx 1.86 P_{\text {TAW }}$, which corresponds to $-q \geq 1.9$, the collision leads to absorption of the TAW by the high-power soliton. A typical example of this outcome is shown for $-q=2.0$, which corresponds to $P_{\text {sol }} \approx 2.01 P_{\text {TAW }}$, in Fig. 12 .

In the above, the outcomes of the strong TAW-soliton interaction, corresponding to the relatively small collision velocity, $c=-0.2$, have been demonstrated. Next, keeping $q=-1.20$, which, as said above, corresponds to $P_{\mathrm{TAW}} \approx$ $P_{\text {sol }}$, i.e., the most complex outcome of the collision, we aim to present results obtained for increasing $|c|$, as the change of the outcome with the variation of the velocity is a relevant issue too. The same outcome as displayed in Fig. 11 for $c=-0.2$, i.e., the formation of the bound Airy-soliton complex, is observed at $|c| \leq 0.3$.

In the interval of velocities $0.3<|c| \leq 1.0$, the velocity is large enough to allow separation of the modes after the collision. In this case, Fig. 13 demonstrates that the interaction, which is still strong enough, leads to a simple result, 


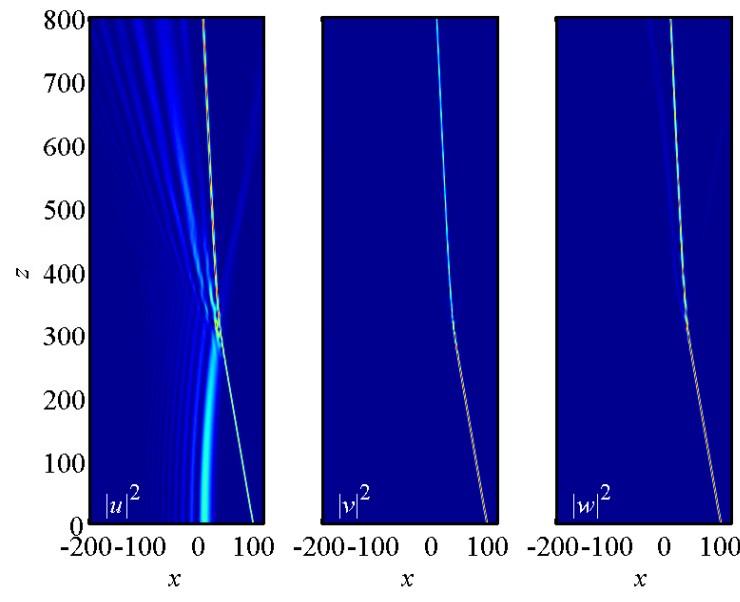

(a)
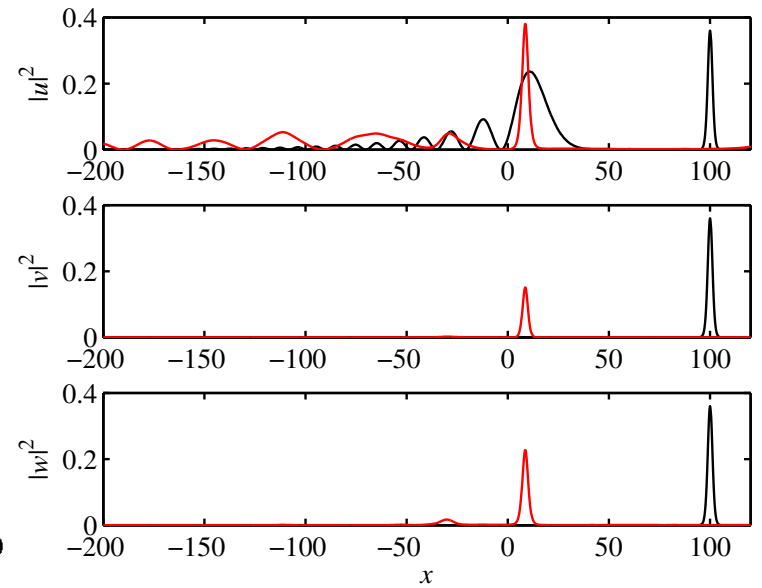

(b)

FIG. 11: (Color online) The same as in Figs. 8 10 but for $q=-1.20$. In this case, the collision between the TAW and soliton with nearly equal total powers creates a bound complex including both waveforms (in a distorted form), which has an almost zero velocity.

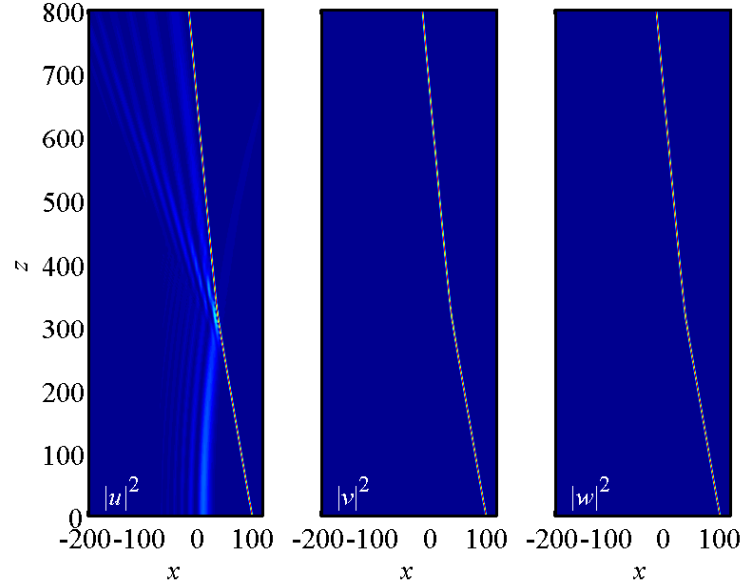

(a)

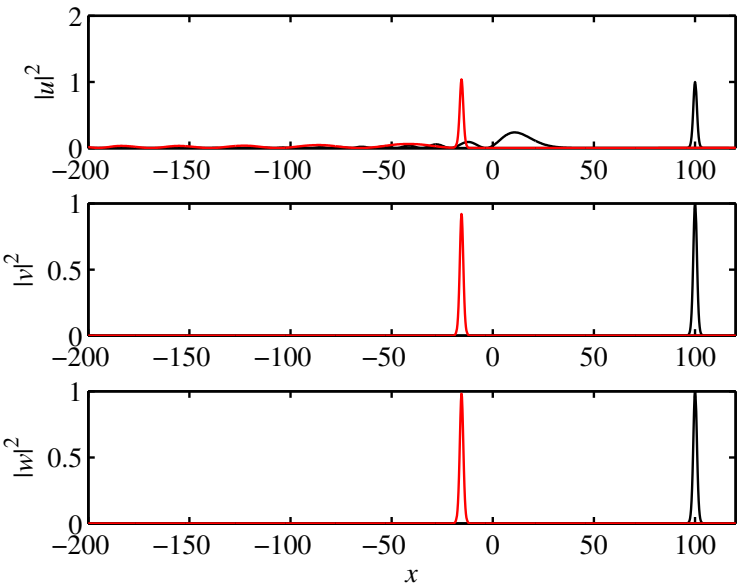

(b)

FIG. 12: (Color online) The same as in Figs. 8.11. but for $q=-2.0$. In this case, the heavy soliton absorbs the TAW with which is collides.

similar to that displayed above in Fig. 9- the transformation of the Airy-soliton pair into a set of two asymmetric solitons, which follow the pre-collision directions of the incident soliton and TAW. It is relevant to note that detailed consideration of the data demonstrates that the increase of the power in the $u$-component of the original soliton, observed in Fig. 11, is explained not by snatching the power from the TAW, but by its transfer from the $v$ - and $w$-components of the same soliton.

In the interval of $1.0<|c| \leq 1.5$, which is represented by Fig. 14 the attenuation of the interaction, caused by the increase of the collision velocity, allows the TAW to partly keep its shape after the collision. Further, at $1.5<|c| \leq 2.5$, the collision begins to feature quasi-elasticity, although the incident soliton is still transformed from the symmetric shape into a conspicuously asymmetric one, see a typical example in Fig. 15 for $c=-2.0$. In this case, like in the situation displayed in Fig. 13, the increase of the $u$-component, observed in its outcome profile, is explained by the transfer of power from the soliton's $v$ - and $w$-components, rather than by capturing power from the original TAW.

Lastly, the collision becomes fully elastic at $|c|>2.5$, as is clearly seen in Fig. $[16$ at $c=-3.0$. Note that the incident symmetric soliton keeps its symmetry in this case.

While the above results were presented for the collisions of the TAW with the moving symmetric solitons, given by 


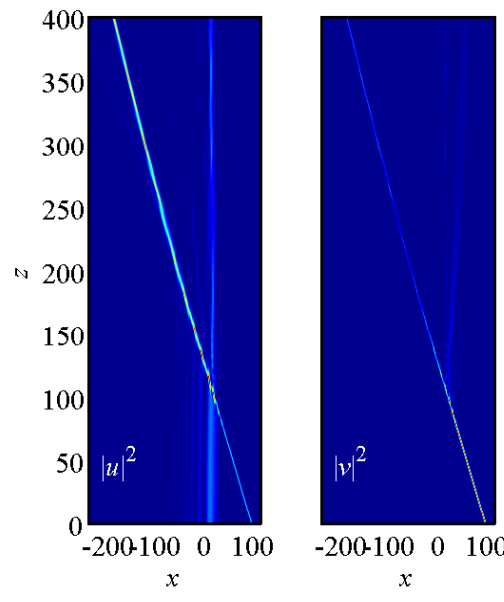

(a)
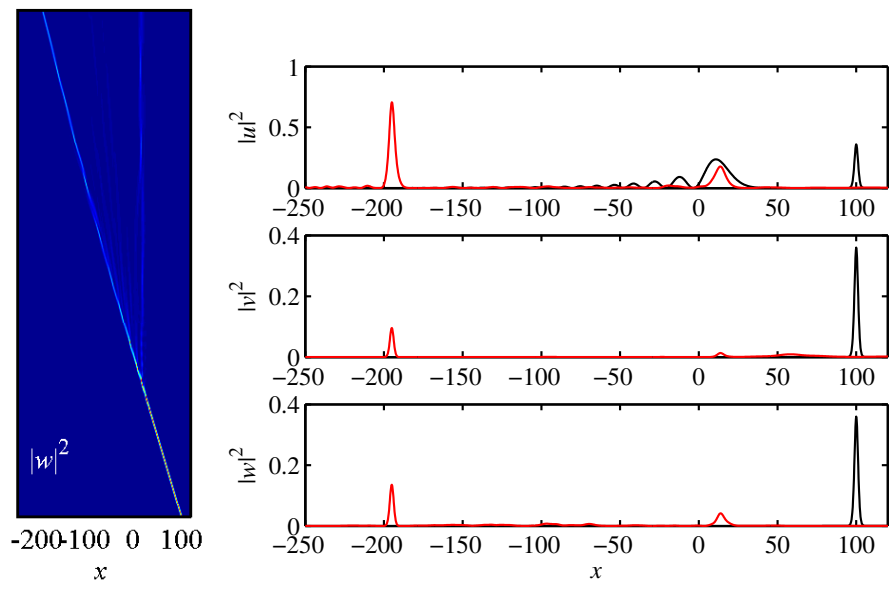

(b)

FIG. 13: (Color online) The same as in Fig. 11, but for the incident soliton with velocity $c=-0.8$. In this case, the TAWsoliton collision transforms the interacting modes into a relatively simple set of two solitons, which continue their motion along the pre-collision directions.

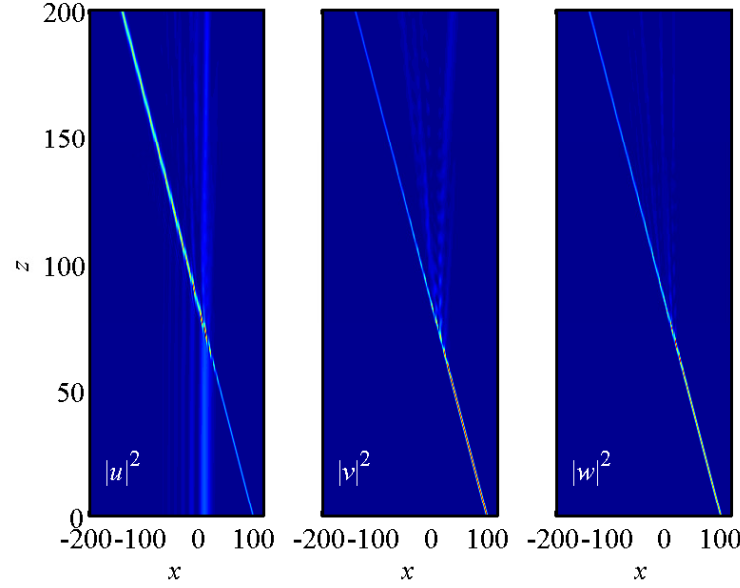

(a)

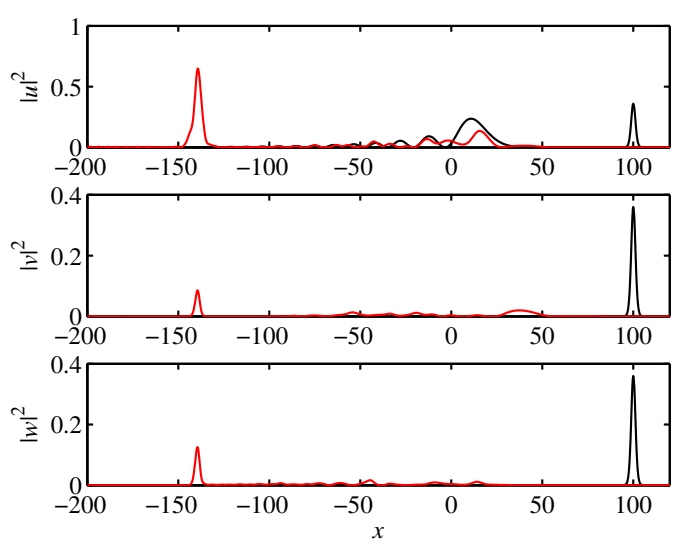

(b)

FIG. 14: (Color online) The same as in Figs. 11 and 13 but for the incident soliton with velocity $c=-1.2$. In this case, the TAW shape partly survives the collision.

exact solution based on Eqs. (13) and (14), collisions of the Airy wave with asymmetric three-wave solitons, such as the one displayed in Fig. 1, have been systematically simulated too (not shown here in detail). It has been concluded that the results are not qualitatively different from those summarized above for the collisions with the symmetric solitons.

\section{CONCLUSION}

The objective of this work is to demonstrate that the generic three-wave system coupled by the $\chi^{(2)}$ interaction makes it possible to consider interactions of the TAWs (truncated Airy waves) and three-wave solitons in the setting which is not available in other nonlinear systems. The advantage of the present system is that the single-wave TAWs, carried by one FF (fundamental-frequency) component, which are not distorted by the nonlinearity, are stable against parametric perturbations (unlike the previously considered setting, with the parametrically unstable TAW in the SH (second-harmonic) component), and the three-wave solitons are stable as well (some additional numerical 


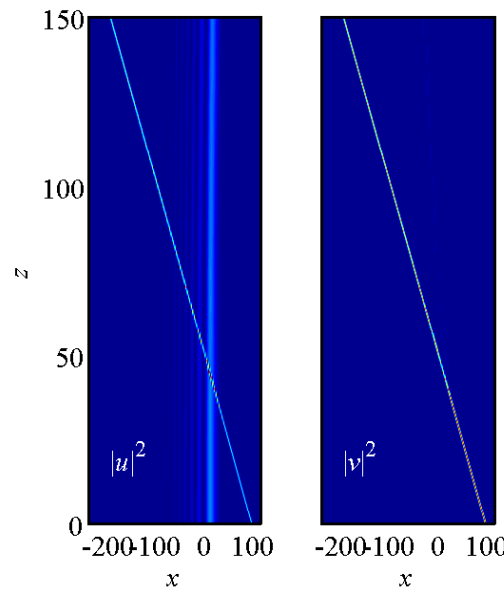

(a)
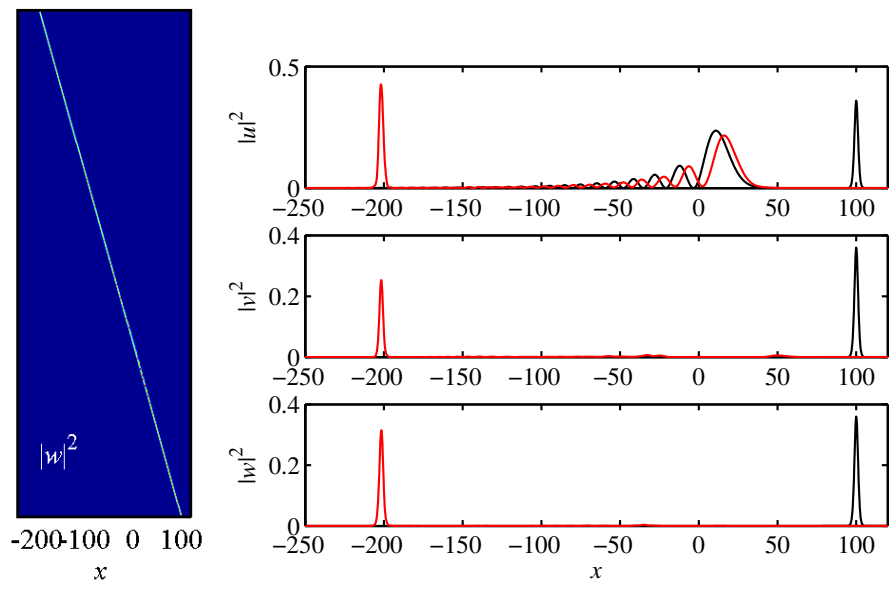

(b)

FIG. 15: (Color online) The same as in Figs. 11, 13, and 14, but for the incident soliton with velocity $c=-2.0$. In this case, the Airy-soliton collision begins to feature quasi-elasticity.

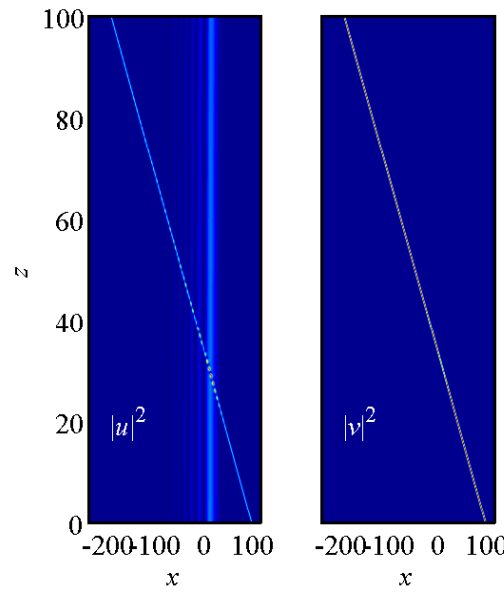

(a)
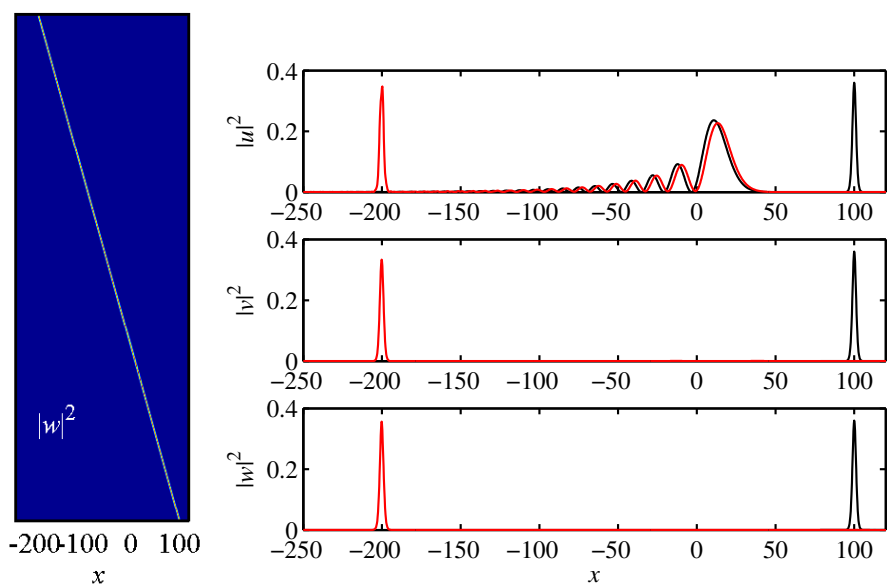

(b)

FIG. 16: (Color online) The same as in Figs 11 and 13 15, but for the incident soliton with velocity $c=-3.0$. In this case, the collision is fully elastic.

and analytical results for these solitons were reported here too). While the TAWs are stable, they are found to be rather fragile against collisions with each other, and with three-wave solitons. In particular, the collision between two mutually symmetric TAWs carried by different FF components leads to the formation of a cluster of solitons, which eventually coagulate into a set of three ones with the increase of the total power. As concerns TAW-soliton collisions, the TAW absorbs incident solitons with a very small power, and a high-power soliton absorbs the TAW. In the intermediate region, the collision breaks the TAW into two solitons (with a remnant TAW attached to one of them), or forms a complex TAW-soliton bound state. The increase of the collision velocity gradually makes the collision quasi-elastic.

As an extension of the present work, it may be quite interesting to develop the analysis for the two-dimensional three-wave system, in which stable single-wave TAWs and three-wave solitons are available too, cf. Ref. [29]. 


\section{ACKNOWLEDGMENT}

This work was supported by the Thailand Research Fund through grant No. RSA5780061.

[1] M. V. Berry and N. L. Balazs, "Non-spreading wave packets", Am. J. Phys. 47, 264-267 (1979).

[2] G. A. Siviloglou and D. N. Christodoulides, "Accelerating finite energy Airy beams", Opt. Lett. 32, 979 (2007).

[3] G. A. Siviloglou, J. Broky, A. Dogariu, and D. N. Christodoulides, "Observation of Accelerating Airy Beams", Phys. Rev. Lett. 99, 213901 (2007).

[4] P. Polynkin, M. Kolesik, J. V. Moloney, G. A. Siviloglou, and D. N. Christodoulides, "Curved plasma channel generation using ultraintense Airy beams", Science 324, 229-232 (2009).

[5] R. El-Ganainy, K. G. Makris, M. A. Miri, D. N. Christodoulides, and Z. Chen, "Discrete beam acceleration in uniform waveguide arrays", Phys. Rev. A 84, 023842 (2011).

[6] P. Rose, F. Diebel, M. Boguslawski, and C. Denz, "Airy beam induced optical routing", Appl. Phys. Lett. 102, 101101 (2013).

[7] R. Driben, Y. Hu, Z. Chen, B. A. Malomed, and R. Morandotti, "Inversion and tight focusing of Airy pulses under the action of third-order dispersion", Opt. Lett. 38, 2499-2501 (2013).

[8] R. Driben, V. V. Konotop, and T. Meier, "Coupled Airy breathers", Opt. Lett. 39, 5523-5526 (2014).

[9] N. K. Efremidis, "Accelerating beam propagation in refractive-index potentials", Phys. Rev. A 98, 023841 (2014).

[10] A. Minovich, A. E. Klein, N. Janunts, T. Pertsch, D. N. Neshev, and Y. S. Kivshar, "Generation and near-field imaging of Airy surface plasmons", Phys. Rev. Lett. 107, 116802 (2011).

[11] L. Li, T. Li, S. M. Wang, C. Zhang, and S. N. Zhu, "Plasmonic Airy Beam Generated by In-Plane Diffraction", Phys. Rev. Lett. 107, 126804 (2011).

[12] I. Epstein and A. Arie, "Arbitrary Bending Plasmonic Light Waves", Phys. Rev. Lett. 112, 023903 (2014).

[13] N. Voloch-Bloch, Y. Lereah, Y. Lilach, A. Gover, and A. Arie, "Generation of electron Airy beams", Nature 494, 331-335 (2013).

[14] N. K. Efremidis, V. Paltoglou, and W. von Klitzig, "Accelerating and abruptly autofocusing matter waves", Phys. Rev. A 87, 043637 (2013).

[15] M. Clerici, Y. Hu, P. Lassonde, C. Milián, A. Couairon, D. N. Christodoulides, Z. Chen, L. Razzari, F. Vidal, F. Légaré, D. Faccio, R. Morandotti, "Laser-assisted guiding of electric discharges around objects", Sci. Adv. 1, e140011 (2015).

[16] S. Fu, Y. Tsur, J. Zhou, L. Shemer, and A. Arie, "Propagation Dynamics of Airy Water-Wave Pulses", Phys. Rev. Lett. 115, 034501 (2015).

[17] I. Kaminer, M. Segev, and D. N. Christodoulides, "Self-accelerating self-trapped optical beams", Phys. Rev. Lett. 106, 213903 (2011).

[18] Y. Fattal, A. Rudnick, and D. M. Marom, "Soliton shedding from Airy pulses in Kerr media", Opt. Exp. 19, 17298-17307 (2011).

[19] A. Lotti, D. Faccio, A. Couairon, D. G. Papazoglou, P. Panagiotopoulos, D. Abdollahpour, and S. Tzortzakis, "Stationary nonlinear Airy beams", Phys. Rev. A 84, 021807(R) (2011).

[20] Y. Hu, Z. Sun, D. Bongiovanni, D. Song, C. Lou, J. Xu, Z. Chen, and R. Morandotti, "Reshaping the trajectory and spectrum of nonlinear Airy beams", Opt. Lett. 37, 3201-3203 (2012).

[21] I. M. Allayarov and E. N. Tsoy, "Dynamics of Airy beams in nonlinear media", Phys. Rev. A 90, 023852 (2014).

[22] C. Ruiz-Jiménez, K. Z. Nóbrega, and M. A. Porras, "On the dynamics of Airy beams in nonlinear media with nonlinear losses", Opt. Exp. 23, 8918-8928 (2015).

[23] L. Zhang, P. Huang, C. Conti, Z. Wang, Y. Hu, D. Lei, Y. Li, and D. Fan, "Decelerating Airy pulse propagation in highly non-instantaneous cubic media", Opt. Exp. 25, 1856-1866 (2017).

[24] T. Ellenbogen, N. Voloch-Bloch, A. Ganany-Padowicz, and A. Arie, "Nonlinear generation and manipulation of Airy beams", Nature Phot. 3, 395-398 (2009).

[25] I. Dolev, T. Ellenbogen, and A. Arie, "Switching the acceleration direction of Airy beams by a nonlinear optical process", Opt. Lett. 35, 1581-1583 (2010).

[26] I. Dolev and A. Arie, "Three wave mixing of Airy beams in a quadratic nonlinear photonic crystals", Appl. Phys. Lett. 97, $171102(2010)$.

[27] I. Dolev, I. Kaminer, A. Shapira, M. Segev, and A. Arie, "Experimental Observation of Self-Accelerating Beams in Quadratic Nonlinear Media", Phys. Rev. Lett. 108, 113803 (2012).

[28] T. Mayteevarunyoo and B. A. Malomed, "Generation of $\chi^{2}$ solitons from the Airy wave through the parametric instability", Opt. Lett. 40, 4947-4950 (2015).

[29] T. Mayteevarunyoo and B. A. Malomed, "Two-dimensional $\chi^{(2)}$ solitons generated by the downconversion of Airy waves", Opt. Lett 41, 2919-2922 (2016).

[30] A. Rudnick and D. M. Marom, "Airy-soliton interactions in Kerr media", Opt. Exp. 19, 25570-25582 (2011).

[31] Y. Zhang, M. R. Belić, H. Zheng, H. Chen, C. Li, Y. Li, and Y. Zhang, "Interactions of Airy beams, nonlinear accelerating beams, and induced solitons in Kerr and saturable nonlinear media", Opt. Exp. 22, 7160-7171 (2013).

[32] Y. Zhang, M. Belić, Z. Wu, H. Zheng, K. Lu, Y. Li, and Y. Zhang, "Soliton pair generation in the interactions of Airy and 
nonlinear accelerating beams", Opt. Lett. 38, 4585-4588 (2013).

[33] N. Wiersma, N. Marsal, M. Sciamanna, and D. Wolfersberger, "Spatiotemporal dynamics of counterpropagating Airy beams", Sci. Rep. 5, 13463 (2015).

[34] M. Shen, J. Gao, and L. Ge, "Solitons shedding from Airy beams and bound states of breathing Airy solitons in nonlocal nonlinear media", Sci. Rep. 5, 9814 (2015).

[35] M. Shen, W. Li, and R.-K. Lee, "Control on the anomalous interactions of Airy beams in nematic liquid crystals", Opt. Exp. 24, 8501 (2016).

[36] C. Hang and G. Huang, "Guiding ultraslow weak-light bullets with Airy beams in a coherent atomic system", Phys. Rev. A 89, 013821 (2014).

[37] G. I. Stegeman, D. J. Hagan, and L. Torner, "Cascading phenomena and their applications to all-optical signal processing, mode-locking, pulse compression and solitons", Opt. Quant. Elect. 28, 1691-1740 (1996).

[38] C. Etrich, F. Lederer, B. A. Malomed, T. Peschel, and U. Peschel, "Optical solitons in media with a quadratic nonlinearity", Prog. Opt. 41, 483-568 (2000).

[39] A. V. Buryak, P. Di Trapani, D. V. Skryabin, and S. Trillo, "Optical solitons due to quadratic nonlinearities: from basic physics to futuristic applications", Phys. Rep. 370, 63-235 (2002).

[40] D. Anderson, M. Lisak, and B. A. Malomed, "Three-wave solitons in a dispersive medium with quadratic nonlinearity", Opt. Commun. 126, 251-254 (1996).

[41] K. Xie, A. D. Boardman, Y. D. Jiang, M. Xie, Y. T. Ye, H. J. Yang, H. M. Jiang, X. C. Yu, J. Xiao, and J. Li, "Stability of non-degenerate parametric soliton in quadratic media", Opt. Commun. 259, 286-292 (2006).

[42] Yu. S. Kivshar and B. A. Malomed. Dynamics of solitons in nearly integrable systems. Rev. Mod. Phys. 61, No. 4, 763-915 (1989). 